\title{
Melhorn, JM and Ackerman, WE (eds): Guides to the Evaluation of Disease and Injury Causation
}

\author{
American Medical Association, United States of America, 2008, 456 pp, \$65.15 \\ (softbound)
}

Bruce A. Barron

Published online: 8 July 2010

(C) Springer Science+Business Media, LLC 2010

Healthcare providers that evaluate or treat injuries and illnesses are frequently asked to opine regarding workplace causation, aggravation, or exacerbation of medical conditions. These types of determinations require an objective review of the pertinent medical information, potential workplace exposures, and alleged mechanisms of injury. Once the medical evidence is gathered, a careful analysis of the data is essential to render an authoritative decision. Most healthcare providers receive very little formal training in disease and injury causality paradigms; however, their opinions are routinely used by administrative entities to determine eligibility for various workers' compensation and disability benefits. Given this potential gap in provider knowledge and training, the publication of Guides to the Evaluation of Disease and Injury Causation (Guides) could not have been more timely. Stated goals of the Guides include assisting the reader in the evaluation of causation and bridging the gap between occupational and non-occupational evidence-based causation.

The Guides were edited and written by nationally recognized experts in the field. The book covers occupational and non-occupational medical conditions and includes numerous case examples which facilitate the learning process. The first seven chapters lay the foundation for causality determinations through detailed discussions of key concepts such as causality, apportionment, aggravation, exacerbation, proximate cause, and producing cause. These initial chapters also include critical information regarding methods for determining work-relatedness, strategies for conducting a causality examination, and a process for writing evidence-based reports. The final 15 chapters provide an evidence-based review of causation for a wide variety of medical conditions such as spinal disorders, upper and low extremity disorders, musculoskeletal disorders, neurological disorders, cardiovascular disorders, and mental illness. All of the major organ systems and many of their associated medical conditions that are frequently encountered in clinical practice are reviewed. The authors' analyses include numerous references that are listed at the end of each chapter.

The Guides is a well-written, well-referenced, and authoritative resource on the topic of injury and disease causation. It is easy to read and understand. The structured guidelines proffered by the Guides are its most important attribute as they allow the provider to readily use and apply them to real-world cases. Although ideally designed for healthcare professionals who must render opinions regarding causality and apportionment, the Guides would also be quite useful to claims managers, case managers, and lawyers who are involved in the administration of benefits or litigation of claims that are based on causality determinations. Guides to the Evaluation of Disease and Injury Causation should be in the tool kit of all professionals engaged in workers' compensation or disability associated claims.

\footnotetext{
B. A. Barron ( $\square)$

Division of Occupational and Environmental Medicine, University of Rochester School of Medicine and Dentistry, Rochester, USA

e-mail: bruce_barron@urmc.rochester.edu
} 